\title{
Full-color tuning in binary polymer:perovskite nanocrystals organic-inorganic hybrid blends
}

\author{
A. Perulli, ${ }^{1}$ A. Balena, ${ }^{1}$ M. Fernandez, ${ }^{1}$ G. Nedelcu, ${ }^{2,3}$ A. Cretí, ${ }^{4}$ M. V. Kovalenko, ${ }^{2,3}$ \\ M. Lomascolo, ${ }^{4}$ and M. Anni ${ }^{1, a)}$ \\ ${ }^{1}$ Dipartimento di Matematica e Fisica "Ennio De Giorgi," Università del Salento, Via per Arnesano, \\ 73100 Lecce, Italy \\ ${ }^{2}$ Institute of Inorganic Chemistry, Department of Chemistry and Applied Bioscience, ETH Zürich, \\ CH-8093 Zürich, Switzerland \\ ${ }^{3}$ Laboratory for Thin Films and Photovoltaics, Empa-Swiss Federal Laboratories for Materials Science \\ and Technology, CH-8600 Dübendorf, Switzerland \\ ${ }^{4}$ IMM-CNR Institute for Microelectronic and Microsystems, Via per Monteroni, 73100 Lecce, Italy
}

(Received 21 December 2017; accepted 6 April 2018; published online 25 April 2018)

\begin{abstract}
The excellent optical and electronic properties of metal halide perovskites recently proposed these materials as interesting active materials for optoelectronic applications. In particular, the high color purity of perovskite colloidal nanocrystals (NCs) had recently motivated their exploration as active materials for light emitting diodes with tunable emission across the visible range. In this work, we investigated the emission properties of binary blends of conjugated polymers and perovskite NCs. We demonstrate that the emission color of the blends is determined by the superposition of the component photoluminescence spectra, allowing color tuning by acting on the blend relative composition. The use of two different polymers, two different perovskite NCs, and different blend compositions is exploited to tune the blend color in the blue-green, yellow-red, and blue-red ranges, including white light generation. Published by AIP Publishing. https://doi.org/10.1063/1.5020201
\end{abstract}

In the field of development of active materials for future optoelectronic applications, lead halide-perovskites are receiving great interest stimulated by the very fast improvement of the performance of optoelectronic devices exploiting perovskites films as active materials. In particular, the efficiency of perovskite solar cells increased from about $3 \%$ up to above $22 \%$ in a few years, ${ }^{1}$ proposing perovskites as one of the most promising classes of materials within the thirdgeneration of solar harvesting materials, including dyes and organic polymers. ${ }^{2,3}$

Moreover, perovskite thin films, either bulk polycrystalline or deposited from nanocrystal (NC) solutions, combine a bandgap tunable by acting on the chemical composition, ${ }^{4,5}$ thus allowing to continuously vary the emission color across visible and near-infrared wavelengths, ${ }^{6}$ with high photoluminescence quantum yields (PLQYs) and good charge mobilities, making perovskite thin films attractive also for light emitting devices, like Light Emitting Diodes (LEDs)., ${ }^{4-11}$

Moreover, perovskite NCs show narrow emission lines, which enable a pure color distribution covering a range greater than the National Television System Committee (NTSC) standard on a Commission International de l'Eclairage (CIE) chromaticity diagram, ${ }^{4,12}$ recently exploited to realize single color LED with external quantum efficiencies up to $6 \% .{ }^{13}$

Despite the promising properties of light emitting perovskites as active materials in LEDs, a particularly important issue to address is the difficulty in obtaining perovskite thin films of uniform thickness and morphology. ${ }^{14}$ In particular, polycrystalline bulk thin films are characterized by pin-holes that can create electrical shunting paths, strongly limiting the

\footnotetext{
${ }^{\text {a) }}$ Author to whom correspondence should be addressed: marco.anni@ unisalento.it
}

electroluminescence yield,${ }^{15}$ and $\mathrm{NC}$ aggregation further worsens the morphology uniformity of thin films deposited from NC solutions. In this frame, it has been recently demonstrated that the combination of a soluble polymer with perovskite precursors can lead to uniform films combining perovskite domains in a polymer matrix, to date exploited for the demonstration of hybrid solar cells ${ }^{16}$ and green emitting LEDs. ${ }^{15}$ Multilayer hybrid organic-perovskite LEDs have also been demonstrated by combining a perovskite layer and a light emitting hole transport polymer. ${ }^{17}$ Concerning perovskite $\mathrm{NCs}$, the dispersion of the materials in organic solvents can be exploited to directly blend the NCs into soluble polymers. This quality has been recently exploited to realize blends between inert polymers and light emitting nanocrystals used as a color conversion layer for white light generation, ${ }^{18,19}$ with the recent demonstration of white generation from a blend between blue emitting nanocrystals and an orange emitting polymer. ${ }^{20}$ Despite these interesting preliminary results, a general investigation of the physics of the interactions between active polymers and perovskite materials is still missing.

In this letter, we present a physical study of the color tunability of hybrid active blends between light emitting polymers (host matrix) and $\mathrm{CsPb}_{3}$ perovskite (guest) NC. We demonstrate that the blend photoluminescence (PL) spectra are mainly determined by the superposition of the constituents, thus allowing color tuning by varying the relative composition of the blend. This result is exploited to continuously tune the blend emission color in three different polymer-NC systems, covering the blue-green, the yellow-red, and the blue-red ranges, including white light generation. Our results propose hybrid polymer-perovskite NC blends as interesting systems for LED applications, combining in a single layer the wide 
emission color tuning of both components, the good film forming properties of the polymers, and the high PLQY of the NCs.

$\mathrm{CsPbBr}_{3}$ and $\mathrm{CsPb}(\mathrm{BrI})_{3} \mathrm{NCs}$ were synthesized as described by Protesescu $\mathrm{et} \mathrm{al} .{ }^{4}$ with some changes as follows: $0.376 \mathrm{mmol} \mathrm{PbBr}_{2}\left[0.188 \mathrm{mmol} \mathrm{PbBr}_{2}\right.$ and $0.188 \mathrm{mmol} \mathrm{PbI}_{2}$ for $\mathrm{CsPb}(\mathrm{BrI})_{3}$, pre-dried oleic acid and oleylamine $[1 \mathrm{ml}$ of each for $\mathrm{CsPBr}_{3}$ and $1.2 \mathrm{ml}$ each for $\mathrm{CsPb}(\mathrm{BrI})_{3}$ ] along with pre-dried 1-octadecene $(5 \mathrm{ml})$ were loaded into a $25 \mathrm{ml} 3$-neck flask and kept for $10 \mathrm{~min}$ at $120^{\circ} \mathrm{C}$ under vigorous stirring. The reaction mixture was switched to a $\mathrm{N}_{2}$ atmosphere and heated up to $180^{\circ} \mathrm{C}$, and $0.8 \mathrm{ml}$ of Cs-oleate was swiftly injected. The reaction was stopped after $10 \mathrm{~s}$ by immersing the flask into a water bath. The obtained NCs were purified by centrifugation (5 $\mathrm{min}$ at $20133 \mathrm{rcf}$ ) and redispersion of the precipitate into $0.5 \mathrm{ml}$ of hexane. The dispersion was centrifuged again, and the precipitate was dispersed in $4 \mathrm{ml}$ of toluene and centrifuged again for $2 \mathrm{~min}$ at $2200 \mathrm{rcf}$. The supernatant was collected, filtrated, and used in our experiments.

Two different polymers have been used, namely, poly(9,9-dioctylfluorene-co-benzothiadiazole) (F8BT, yellow emitting) and poly(9,9-dioctylfluorene) (PFO, blue emitting), both obtained from ADSdyes and used as received. The blends were realized by mixing at different weight ratios a polymer solution to a $\mathrm{NC}$ solution, both in toluene and with a concentration of $10 \mathrm{mg} / \mathrm{ml}$. The relative concentration has been converted into percentage of emitters by considering the different molecular weights of the polymers and the NCs. The films were obtained by drop casting on glass substrates pre-cleaned with toluene, thus obtaining films with a typical thickness of about $300 \mathrm{~nm} \pm 30 \mathrm{~nm}$.

The PL measurements were performed by exciting the samples with a MNL-100 Nitrogen Laser (Lasertechnik Berlin) at a wavelength of $337 \mathrm{~nm}$, with an average power density of about $1.3 \mathrm{~W} / \mathrm{cm}^{2}$, collecting the PL signal in backscattering configuration by an optical fiber connected to a spectrometer (Acton Research Corporation Spectra Pro-750) coupled to a Peltier cooled CCD. The time resolved PL (TR-PL) measurements were performed by exciting the samples by a solid state pulsed laser (mod. PLP-10, Hamamatsu), which provides pulse at a wavelength of $400 \mathrm{~nm}$ of about $58 \mathrm{ps}$ at a repetition rate of $1 \mathrm{MHz}$. The PL has been dispersed using an iHR320 (focal length of $0.32 \mathrm{~m}$ ) Horiba monochromator equipped with a Peltier cooled Hamamatsu photomultiplier (Head-on mod R943-02), operating in single photon counting. The TimeCorrelated Single Photon Counting (TCSPC) technique has been used to record TR-PL in the spectral visible range by using an Edinburg Instruments TCC900 TSCPC electronics card. The temporal resolution of the system is about $0.46 \mathrm{~ns}$.

In order to investigate the general emission properties of combinations of polymers and perovskite NCs we realized three different blends:

1. Blend between the blue polymer (PFO) and green emitting $\mathrm{NCs}\left(\mathrm{CsPbBr}_{3}, \mathrm{GNC}\right.$ in the following).

2. Blend between the yellow polymer (F8BT) and red emitting $\mathrm{NCs}\left(\mathrm{CsPb}_{3}, \mathrm{X}=\mathrm{Br} / \mathrm{I}, \mathrm{RNC}\right.$ in the following).

3. Blend between PFO and RNC.

The PL spectra of the individual components of the blend and the resulting spectrum of the blend of F8BT:NCR with a percentage composition of $2.1 \%$ are reported in Fig. 1. The PL spectrum clearly shows the presence of two distinct peaks related to the emission of F8BT $\left(\lambda_{F 8 B T}\right.$ $\approx 547 \mathrm{~nm})$ and to the emission of the NCs $\left(\lambda_{R N C} \approx 592 \mathrm{~nm}\right)$. The RNC peak in the blend is slightly blue-shifted with respect to that of the RNC neat film, likely due to the suppression of energy migration within the NC films, due to the dilution in the polymer matrix. ${ }^{21}$

It is interesting to observe that in fully organic hostguest blends, the presence of a host emitting in the spectral range of the guest absorption typically leads to efficient Förster resonant energy transfer (FRET) from the host to the guest, strongly suppressing the host PL already for guest contents of few percent. ${ }^{22}$ In our case, the blend PL spectrum shows instead strong polymer emissions, despite the good spectral overlap between the polymer PL and the $\mathrm{CsPbX}_{3}$ absorption, strongly suggesting the lack of efficient FRET from the polymer to the NCs.

In order to investigate the eventual presence of energy transfer from the F8BT to the NCs and its efficiency, we performed TR-PL measurements at the F8BT peak wavelength for pure F8BT and two blends with different $\mathrm{NC}$ relative contents, namely, $2.1 \%$ and $8.0 \%$. The obtained results (see Fig. 2) clearly show a PL decay becoming faster as the nanocrystal content increases. The pure F8BT relaxation dynamics is monoexponential with a best fit lifetime of $1.84 \pm 0.03 \mathrm{~ns}$. When the NCs are added to the film, a biexponential decay is observed for a NC content of $2.1 \%$, with a dominant (relative amplitude of about 90\%) fast component with a lifetime of $820 \pm 50$ ps and an unaffected long lifetime, and a faster monoexponential dynamic for a NC content of $8.0 \%$, with a lifetime of $677 \pm 7$ ps.

This result clearly evidences the presence of a further F8BT exciton relaxation channel when the NCs are added, whose importance increases with the NC content. The lifetime $\tau^{\prime}$ of this process can be determined assuming that the intrinsic F8BT lifetime $\tau_{B T}$ is the same in all the samples and remembering that the total lifetime $\tau_{P L}$ is given by $1 / \tau_{P L}=1 / \tau_{B T}$ $+1 / \tau^{\prime}$. By using the best fit values and uncertainty of all the

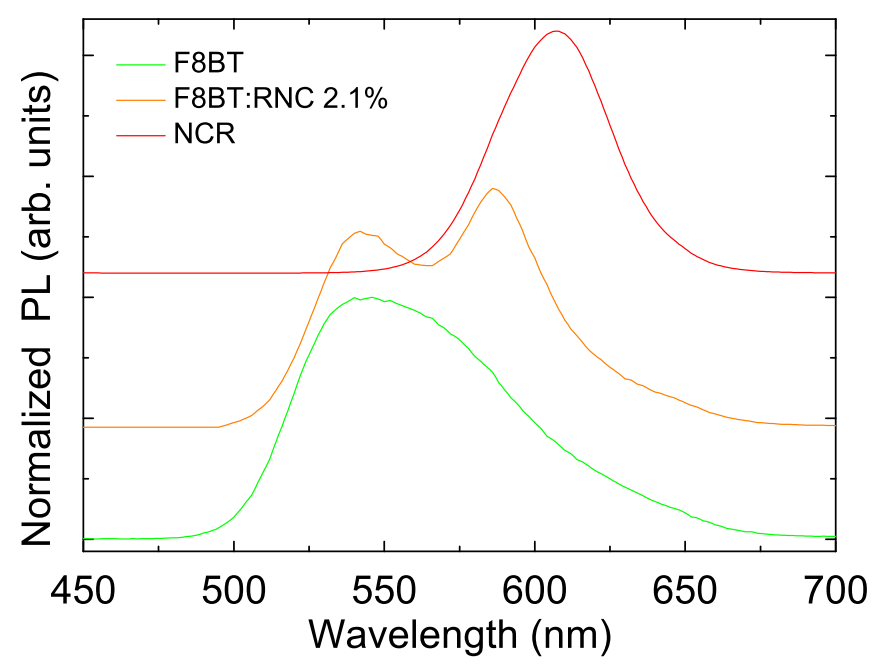

FIG. 1. PL spectra of F8BT (bottom), RNC (top), and the F8BT:RNC blend (middle) with $2.1 \%$ of NCs by weight. The spectra are normalized to the peak value and vertically translated for clarity. The blend spectrum clearly shows the superposition of the spectra of the component materials. 


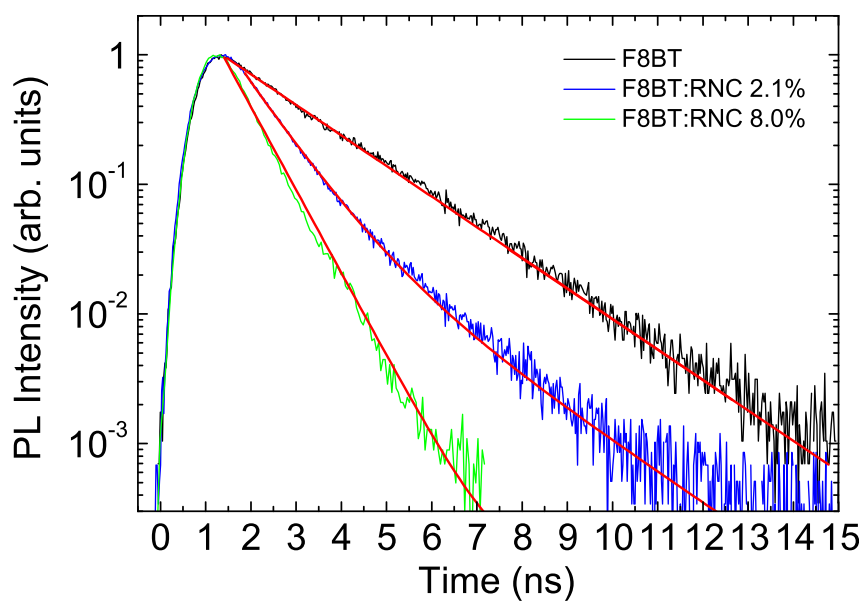

FIG. 2. PL relaxation dynamic at the F8BT peak wavelength for the F8BT:RNC blends, evidencing the lifetime shortening as the NC content increases. The red lines are the best fit curves.

parameters, we estimate $\tau^{\prime}=1.48 \pm 0.17 \mathrm{~ns}$ and $\tau^{\prime}=1.07$ $\pm 0.03 \mathrm{~ns}$, for $\mathrm{NC}$ contents of $2.1 \%$ and $8.0 \%$, respectively. In order to determine the origin of this process, we quantitatively investigated the role of Förster Transfer from F8BT to the $\mathrm{NCs}$, starting from the estimate of the Förster radius ${ }^{23}$

$$
R_{0}^{6}=\frac{9000 k^{2} \log 10}{128 \pi^{5} n^{4} N_{A V}} \Phi_{d} \int_{0}^{\infty} \frac{E_{d}(\tilde{\nu}) \epsilon_{a}(\tilde{\nu})}{\tilde{\nu}^{4}} d \tilde{\nu},
$$

where $k^{2}$ is an orientation factor which for random relative orientation of the donor and acceptor dipoles is $2 / 3, N_{A V}$ is the Avogadro number, $\Phi_{d}$ is the donor emission quantum efficiency, $\tilde{\nu}$ is the wavenumber in $\mathrm{cm}^{-1}, E_{d}(\tilde{\nu})$ is the normalized donor emission spectrum, and $\epsilon_{a}(\tilde{\nu})$ is the molar decadic extinction coefficient of the acceptor. A Förster radius of $67.8 \AA$ has been determined from the experimental spectra, by assuming $n \approx 1.8$ (Ref. 24), $\Phi_{d}=0.5$ (Ref. 25), and $\epsilon_{a}=1.36 \times 10^{7} \mathrm{M}^{-1} \mathrm{~cm}^{-1}$ at the wavenumber of the first absorption resonance, as determined from absorption measurements as a function of the solution concentration. For the sake of comparison, we observe that the obtained $\mathrm{R}_{0}$ value is about $20 \%$ larger than the value reported for polymer-CdSe/ $\mathrm{ZnS}$ nanocrystal blends, ${ }^{26}$ resulting in a FRET rate (depending on $\mathrm{R}_{0}^{6}$ ) about 3 times larger (at a fixed donor lifetime and donor acceptor distance, see supplementary material). We then estimated a lower limit of the total FRET lifetime by a volume integral of the rate donor-acceptor distance dependence (see supplementary material), obtaining $3.5 \mathrm{~ns}$ and $0.9 \mathrm{~ns}$ for NC contents of $2.1 \%$ and $8.0 \%$, respectively.

For a NC content of $2.1 \%$, the FRET lifetime is about 2.4 times larger than $\tau^{\prime}$, while for the $8.0 \%$ blend, the two times are comparable. Our data thus suggest that the experimental values of the polymer-NC interaction time are likely shorter than the FRET lifetime, suggesting that the main polymer-NC interaction process is not given by FRET. A further possible contribution to the F8BT lifetime reduction could be direct electron and hole transfer by Dexter transfer, which would be energetically possible as the conductionband minimum $(\mathrm{CBm})$ and the valence-band maximum (VBM) of RNC are sandwiched between the lowest unoccupied molecular orbital (LUMO) and the highest occupied

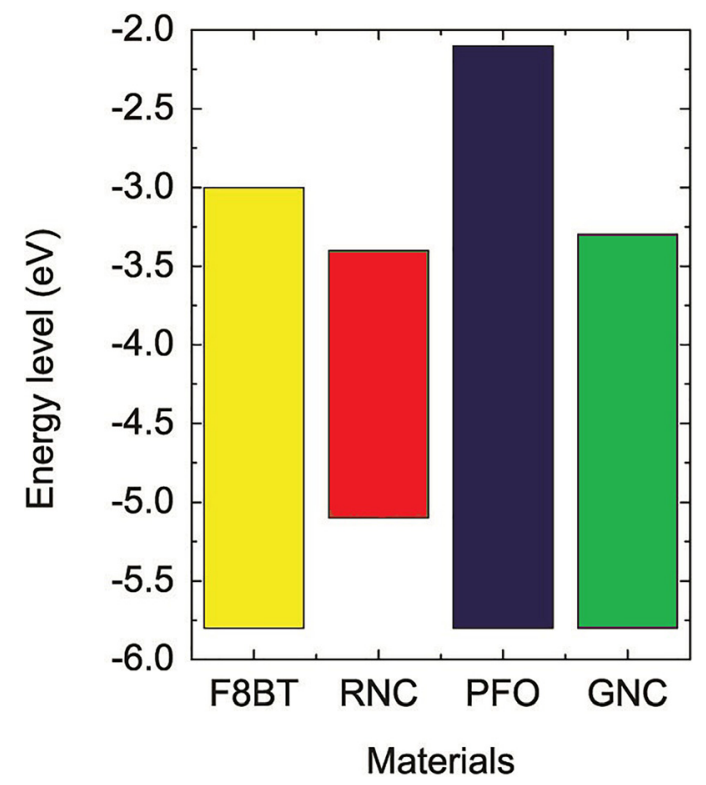

FIG. 3. Band edge energies of F8BT, RNC, PFO, and GNC, evidencing that the NC top valence and bottom conduction energy levels are between the HOMO and LUMO levels of the polymers.

molecular orbital (HOMO) of F8BT (see Fig. 3). ${ }^{27,28}$ Despite this, we observe that Dexter transfer typically dominates over FRET for the donor-acceptor distance below $1 \mathrm{~nm},{ }^{29}$ which is likely forbidden in our case by the presence of the ligands on the NC surface. Overall, this analysis evidences that a clear interaction between the polymer and NC is present in the blend, which anyway cannot be fully explained by the eventual excitation transfer from the polymer to the NCs. Further experiments will be necessary to determine the origin of the polymer-NC interaction.

We finally calculated the blend color coordinates according to the standards of the Commission International de l'Eclairage (CIE), which lie along the line joining the RNC and the F8BT coordinates (see Fig. 4), as expected for additive color synthesis.

Similar results are observed for the PFO:GNC blend (see Fig. 5), in which we clearly observe the superposition of

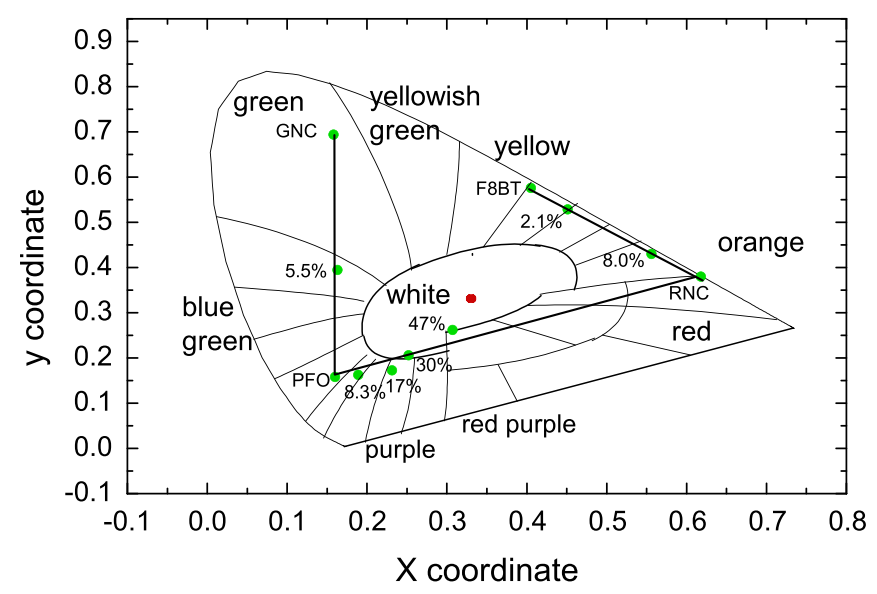

FIG. 4. CIE diagram with the color coordinates of the explored materials and blends. The lines connect the two materials composing the different blends. The percentage numbers indicate the relative NC content in the blend. It is evident that the blend color coordinates are along the lines connecting the component material ones. 


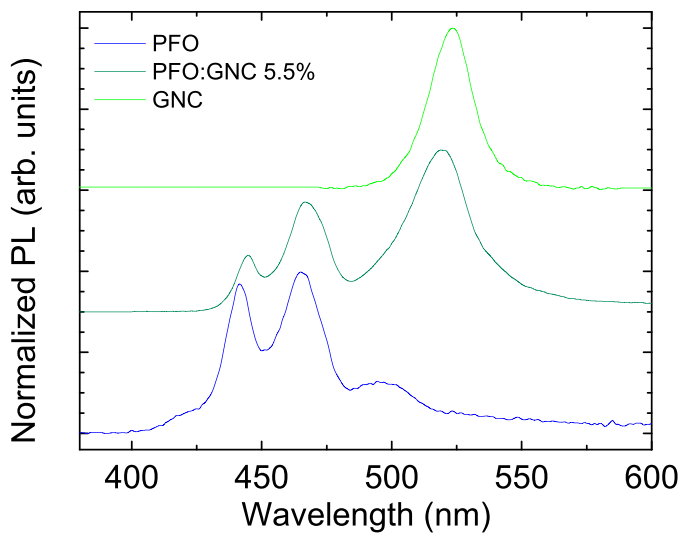

FIG. 5. PL spectra of PFO (bottom), GNC (top), and the PFO:GNC blend (middle) with a NC content of $5.5 \%$. The spectra are normalized to the peak value and vertically translated for clarity.

two peaks due to PFO at about 445 and $467 \mathrm{~nm}$ and a further peak near $520 \mathrm{~nm}$ due to the GNC photoluminescence, again slightly blue-shifted with respect to the PL peak of pure GNC. As for the first blend, the CIE coordinates of the PFO:GNC blend are positioned on the line joining GNC and PFO coordinates (see Fig. 4).

We then exploited these preliminary results in order to realize a blend between two materials, potentially allowing white light generation, namely, PFO and RNC, by realizing four different blends with a NC content between $8 \%$ and $47 \%$.

The PL spectra of these blends (see Fig. 6) clearly show the superposition of the PFO blue PL with the red NC PL, with a progressive increase in the NC PL contribution to the

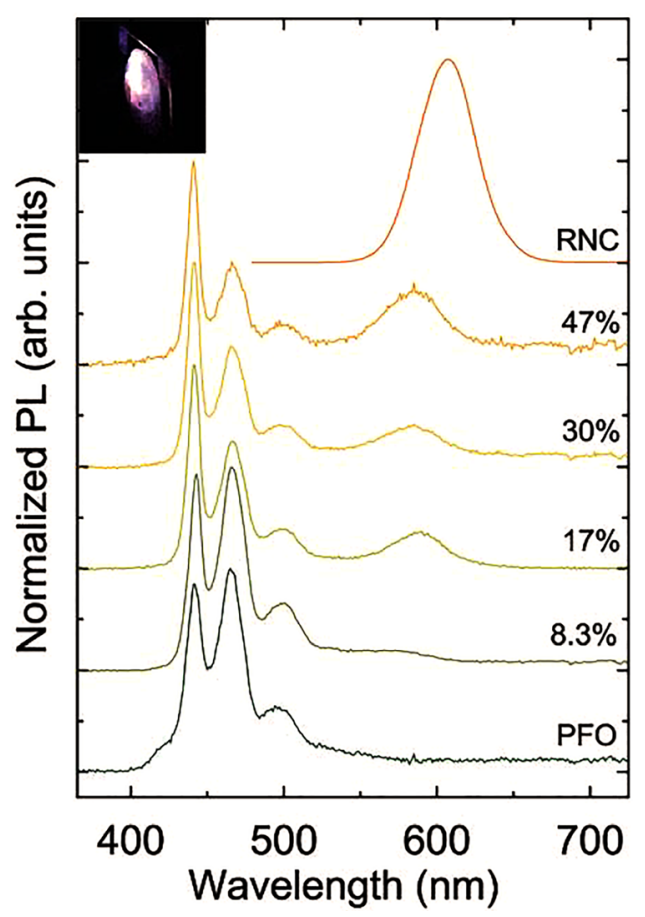

FIG. 6. PL emission spectra of PFO (bottom), RNC (top), and the blend $\mathrm{PFO}$ RNC at four different relative concentrations. The relative NC content increases from bottom to top. The spectra are normalized to the peak value and vertically translated for clarity. The progressive increase in the NC PL relative contribution as the NC content increases is well visible. Inset: photograph of the white emitting blend. blend spectrum as the $\mathrm{NC}$ content is increased, resulting in a progressive color variation from blue to red.

The CIE coordinates of PFO:RNC blends are positioned on the line joining $\mathrm{PFO}$ and $\mathrm{RNC}$, passing through the white spectral region (see Fig. 4). In particular, white emission is observed from the blend with a percentage of $47 \%$ of RNC (see the inset of Fig. 6).

The quality of the generated white light can be easily improved either by a proper variation of the halide composition in the NCs ( $\mathrm{Br}$ and I), in order to move the emission of $\mathrm{RNC}$ to lower wavelength (around $580 \mathrm{~nm}$ instead of $592 \mathrm{~nm}$ ), or by using a polymer host with an emission in the blue-green range. ${ }^{18}$

In conclusion, we have demonstrated the possibility of achieving full color tunability in binary hybrid blends between conjugated polymers and perovskite $\mathrm{NC}\left(\mathrm{CsPb}_{3}\right)$, which combine easy deposition from solution, the good film forming properties of the polymers, and the good emission properties of both blend components. Considering the wide family of available light emitting polymers and the development of OLEDs up to commercial applications, polymer:perovskite NC hybrid blends are potentially extremely interesting active materials for broadly tunable LEDs, allowing the generation of virtually any required specific color, including white, by properly choosing the active materials and the blend content.

See supplementary material for the results of NC shapes and PLQY characterization and the details of the calculation of the total FRET lifetime starting from the Förster Radius.

M.K. acknowledges financial support from the European Union through FP7 (ERC Starting Grant NANOSOLID, GA No. 306733).

\footnotetext{
${ }^{1}$ National Renewable Energy Laboratory, https://www.nrel.gov/pv/assets/ images/efficiency-chart.png for "Research Cell Record Efficiency Chart" (last accessed March 9, 2018).

${ }^{2}$ B. E. Hardin, H. J. Snaith, and M. D. McGehee, Nat. Photonics 6, 162 (2012).

${ }^{3}$ G. Li, R. Zhu, and Y. Yang, Nat. Photonics 6, 153 (2012).

${ }^{4}$ L. Protesescu, S. Yakunin, M. I. Bodnarchuk, F. Krieg, R. Caputo, C. H. Hendon, R. X. Yang, A. Walsh, and M. V. Kovalenko, Nano Lett. 15, 3692 (2015).

${ }^{5}$ G. Nedelcu, L. Protesescu, S. Yakunin, M. Bodnarchuk, M. Grotevent, and M. Kovalenko, Nano Lett. 15, 5635 (2015).

${ }^{6}$ N. Kitazawa, Y. Watanabe, and Y. Nakamura, J. Mater. Sci. 37, 3585 (2002).

${ }^{7}$ F. Deschler, M. Price, S. Pathak, L. E. Klintberg, D. D. Jarausch, R. Higler, S. Hüttner, T. Leijtens, S. D. Stranks, H. J. Snaith, M. Atatüre, R. T. Phillips, and R. H. Friend, J. Phys. Chem. Lett. 5, 1421 (2014).

${ }^{8}$ Z. K. Tan, R. S. Moghaddam, M. L. Lai, P. Docampo, R. Higler, F. Deschler, M. Price, A. Sadhanala, L. M. Pazos, D. Credgington, F. Hanusch, T. Bein, H. J. Snaith, and R. H. Friend, Nat. Nanotechnol. 9, 687 (2014).

${ }^{9}$ N. J. Jeon, J. H. Noh, W. S. Yang, Y. C. Kim, S. Ryu, J. Seo, and S. I. Seok, Nature 517, 476 (2015).

${ }^{10}$ S. Yakunin, L. Protesescu, F. Krieg, M. I. Bodnarchuk, G. Nedelcu, M. Humer, G. D. Luca, M. Fiebig, W. Heiss, and M. V. Kovalenko, Nat. Commun. 6(1), 8056 (2015).

${ }^{11}$ L. Protesescu, S. Yakunin, S. Kumar, J. Bar, F. Bertolotti, N. Masciocchi, A. Guagliardi, M. Grotevent, I. Shorubalko, M. I. Bodnarchuk, C.-J. Shih, and M. V. Kovalenko, ACS Nano 11, 3119 (2017).

${ }^{12}$ B. R. Sutherland and E. H. Sargent, Nat. Photonics 10, 295 (2016).
} 
${ }^{13}$ G. Li, F. Wisnivesky, R. Rivarola, N. J. L. K. Davis, S. Bai, T. C. Jellicoe, F. de la Peña, S. Hou, C. Ducati, F. Gao, R. H. Friend, N. C. Greenham, and Z.-K. Tan, Adv. Mater. 28, 3528 (2016).

${ }^{14}$ Y. Ling, Z. Yuan, Y. Tian, X. Wang, J. C. Wang, Y. Xin, K. Hanson, B. Ma, and H. Gao, Adv. Mater. 28, 305 (2016).

${ }^{15}$ G. Li, Z.-K. Tan, D. Di, M. L. Lai, L. Jiang, J. H.-W. Lim, R. H. Friend, and N. C. Greenham, Nano Lett. 15, 2640 (2015).

${ }^{16}$ S. Masi, S. Colella, A. Listorti, V. Roiati, A. Liscio, V. Palermo, A. Rizzo, and G. Gigli, Sci. Rep. 5, 7725 (2015).

${ }^{17}$ A. Sadhanala, A. Kumar, S. Pathak, A. Rao, U. Steiner, N. C. Greenham, H. J. Snaith, and R. H. Friend, Adv. Electron. Mater. 1, 1500008 (2015).

${ }^{18}$ S. Pathak, N. Sakai, F. W. R. Rivarola, S. D. Stranks, J. Liu, G. E. Eperon, C. Ducati, K. Wojciechowski, J. T. Griffiths, A. A. Haghighirad, A. Pellaroque, R. H. Friend, and H. J. Snaith, Chem. Mater. 27, 8066 (2015).

${ }^{19}$ M. Meyns, M. Peralvarez, A. Heuer-Jungemann, W. Hertog, M. Ibanez, R. Nafria, A. Genc, J. Arbiol, M. V. Kovalenko, J. Carreras, A. Cabot, and A. G. Kanaras, Appl. Mater. Interfaces 8, 19579 (2016).
${ }^{20}$ E.-P. Yao, Z. Yang, L. Meng, P. Sun, S. Dong, Y. Yang, and Y. Yang, Adv. Mater. 29, 1606859 (2017).

${ }^{21}$ C. de Weerd, L. Gomez, H. Zhang, W. J. Buma, G. Nedelcu, M. V. Kovalenko, and T. Gregorkiewicz, J. Phys. Chem. C 120, 13310 (2016).

${ }^{22}$ A. Camposeo, E. Mele, L. Persano, D. Pisignano, and R. Cingolani, Phys. Rev. B 73, 165201 (2006).

${ }^{23}$ T. Förster, Discuss. Faraday Soc. 27, 7 (1959).

${ }^{24}$ M. Campoy-Quiles, G. Heliotis, R. Xia, M. Ariu, M. Pintani, P. Etchegoin, and D. Bradley, Adv. Funct. Mater. 15, 925 (2005).

${ }^{25}$ P. Herguth, X. Jiang, M. S. Liu, and A. K.-Y. Jen, Macromolecules 35, 6094 (2002).

${ }^{26}$ M. Anni, L. Manna, R. Cingolani, D. Valerini, A. Cretí, and M. Lomascolo, Appl. Phys. Lett. 85, 4169 (2004).

${ }^{27}$ V. K. Ravi, G. B. Markad, and A. Nag, ACS Energy Lett. 1, 665 (2016).

${ }^{28}$ J. S. Park, B. R. Lee, E. Jeong, H.-J. Lee, J. M. Lee, J.-S. Kim, J. Y. Kim, and H. Young, Appl. Phys. Lett. 99, 163305 (2011).

${ }^{29}$ A. R. S. Kandada, G. Grancini, A. Petrozza, S. Perissinotto, D. Fazzi, S. S. K. Raavi, and G. Lanzani, Sci. Rep. 3, 2073 (2013). 